\title{
UNBOUNDED TOWERS AND PRODUCTS
}

\author{
PIOTR SZEWCZAK AND MAGDALENA WŁUDECKA
}

\begin{abstract}
We investigate products of sets of reals with combinatorial covering properties. A topological space satisfies $S_{1}(\Gamma, \Gamma)$ if for each sequence of point-cofinite open covers of the space, one can pick one element from each cover and obtain a point-cofinite cover of the space. We prove that, if there is an unbounded tower, then there is a nontrivial set of reals satisfying $S_{1}(\Gamma, \Gamma)$ in all finite powers. In contrast to earlier results, our proof does not require any additional set-theoretic assumptions.

A topological space satisfies $\left(\begin{array}{l}\Omega \\ \Gamma\end{array}\right)$ (also known as Gerlits-Nagy's property $\gamma$ ) if every open cover of the space such that each finite subset of the space is contained in a member of the cover, contains a point-cofinite cover of the space. We investigate products of sets satisfying $\left(\begin{array}{l}\Omega \\ \Gamma\end{array}\right)$ and their relations to other classic combinatorial covering properties. We show that finite products of sets with a certain combinatorial structure satisfy $\left(\begin{array}{l}\Omega \\ \Gamma\end{array}\right)$ and give necessary and sufficient conditions when these sets are productively $(\underset{\Gamma}{\Omega})$.
\end{abstract}

\section{INTRODUCTION}

Let $\mathbb{N}$ be the set of natural numbers and $[\mathbb{N}]^{\infty}$ be the set of infinite subsets of $\mathbb{N}$. In a natural way an element of $[\mathbb{N}]^{\infty}$ can be viewed as an increasing function in $\mathbb{N}^{\mathbb{N}}$. A subset of $[\mathbb{N}]^{\infty}$ is unbounded if for each element $a \in[\mathbb{N}]^{\infty}$, there is an element $b$ in the set such that the set $\{n: a(n)>b(n)\}$ is infinite. A set $a$ is an almost subset of a set $b$, denoted $a \subseteq^{*} b$, if the set $a \backslash b$ is finite.

Definition 1.1. Let $\kappa$ be an uncountable ordinal number. A set $\left\{x_{\alpha}: \alpha<\kappa\right\} \subseteq[\mathbb{N}]^{\infty}$ is a $\kappa$-unbounded tower if it is unbounded and, for all ordinal numbers $\alpha, \beta<\kappa$ with $\alpha<\beta$, we have $x_{\alpha}{ }^{*} \supseteq x_{\beta}$.

For an uncountable ordinal number $\kappa$, the existence of a $\kappa$-unbounded tower is independent of ZFC [15]. It turns out that such a set is a significant object used in constructions nontrivial sets with combinatorial covering properties.

By space we mean a topological space. A cover of a space is a family of proper subsets of the space whose union is the entire space, and an open cover is a cover whose members are open subsets of the space. A $\gamma$-cover of a space is an infinite cover such that each point of the space belongs to all but finitely many sets of the cover. For a space $X$, let $\Gamma(X)$ be the family of all open $\gamma$-covers of the space $X$. A space $X$ satisfies $S_{1}(\Gamma, \Gamma)$ if for each sequence $\mathcal{U}_{1}, \mathcal{U}_{2}, \ldots \in \Gamma(X)$, there are sets $U_{1} \in \mathcal{U}_{1}, U_{2} \in \mathcal{U}_{2}, \ldots$ such that $\left\{U_{n}: n \in \mathbb{N}\right\} \in \Gamma(X)$. This property was introduced by Scheepers [23] and it was studied in the context of local properties of functions spaces [24, 25].

A set of reals is a space homeomorphic to a subset of the real line. We restrict our consideration to the realm of sets of reals. We identify the family $\mathrm{P}(\mathbb{N})$ of all subsets of the

2010 Mathematics Subject Classification. 26A03, 54D20, 03E75, 03E17.

Key words and phrases. unbounded tower, $\mathrm{S}_{1}(\Gamma, \Gamma)$, Gerlits-Nagy, $\gamma$-property, $\gamma$-set, selection principles, products. 
set $\mathbb{N}$ with the Cantor space $\{0,1\}^{\mathbb{N}}$, via characteristic functions. Since the Cantor space is homeomorphic to Cantor's set, every subspace of the space $\mathrm{P}(\mathbb{N})$ is considered as a set of reals. Let Fin be the family of all finite subsets of $\mathbb{N}$. Let $\kappa$ be an uncountable ordinal number. A set $X \cup$ Fin is a $\kappa$-unbounded tower set, if the set $X$ is a $\kappa$-unbounded tower in $[\mathbb{N}]^{\infty}$. Let $\mathfrak{b}$ be the minimal cardinality of an unbounded subset of $[\mathbb{N}]^{\infty}$ Each $\mathfrak{b}$-unbounded tower set satisfies $\mathrm{S}_{1}(\Gamma, \Gamma)([25$, Theorem 6], [15, Proposition 2.5]). Miller and Tsaban proved that, assuming some additional set-theoretic assumption, each $\mathfrak{b}$-unbounded tower set satisfies $S_{1}(\Gamma, \Gamma)$ in all finite powers [15, Theorem 2.8]. We prove that the same statement remains true with no extra assumption used by Miller and Tsaban. The proof method is new.

An $\omega$-cover of a space is an open cover such that, each finite subset of the space is contained in a set from the cover. A space satisfies $\left(\begin{array}{l}\Omega \\ \Gamma\end{array}\right)$ if every $\omega$-cover of the space contains a $\gamma$-cover of the space. This property was introduced by Gerlits and Nagy [9]. A pseudointersection of a family of infinite sets is an infinite set $a$ with $a \subseteq^{*} b$ for all sets $b$ in the family. A family of infinite sets is centered if the finite intersections of its elements, are infinite. Let $\mathfrak{p}$ be the minimal cardinality of a subfamily of $[\mathbb{N}]^{\infty}$ that is centered and has no pseudointersection. By the result of Orenshtein and Tsaban ([17, Theorem 3.6], [18, Theorem 6]), each p-unbounded tower set satisfies $\left(\begin{array}{c}\Omega \\ \Gamma\end{array}\right)$. A set is productively $\left(\begin{array}{c}\Omega \\ \Gamma\end{array}\right)$ if its product space with any set satisfying $\left(\begin{array}{c}\Omega \\ \Gamma\end{array}\right)$, satisfies $\left(\begin{array}{c}\Omega \\ \Gamma\end{array}\right)$. Miller, Tsaban and Zdomskyy proved that, each $\omega_{1}$-unbounded tower set is productively $\left(\begin{array}{l}\Omega \\ \Gamma\end{array}\right)$ [16, Theorem 2.8]. We show that, if each set of cardinality less than $\mathfrak{p}$ is productively $\left(\begin{array}{l}\Omega \\ \Gamma\end{array}\right)$, then each $\mathfrak{p}$-unbounded tower set is productively $\left(\begin{array}{l}\Omega \\ \Gamma\end{array}\right)$. Moreover, the product space of finitely many $\mathfrak{p}$-unbounded tower sets, satisfies $\left(\begin{array}{l}\Omega \\ \Gamma\end{array}\right)$. We also consider products of sets satisfying $\left(\begin{array}{c}\Omega \\ \Gamma\end{array}\right)$ and their relations to classic combinatorial covering properties.

\section{Generalized TOWERS}

We generalize the notion of $\kappa$-unbounded tower for an uncountable ordinal number $\kappa$. Let $n, m$ be natural numbers with $n<m$. Define $[n, m):=\{i \in \mathbb{N}: n \leq i<m\}$. A set $x \in[\mathbb{N}]^{\infty}$ omits the interval $[n, m)$, if $x \cap[n, m)=\emptyset$.

Lemma 2.1 (Folklore [29, Lemma 2.13]). A set $X \subseteq[\mathbb{N}]^{\infty}$ is unbounded if and only if for each function $a \in[\mathbb{N}]^{\infty}$, there is a set $x \in X$ that omits infinitely many intervals $[a(n), a(n+$ $1)$ ).

Let $\kappa$ be an uncountable ordinal number, and $\left\{x_{\alpha}: \alpha<\kappa\right\}$ be a $\kappa$-unbounded tower in $[\mathbb{N}]^{\infty}$. Fix a function $a \in[\mathbb{N}]^{\infty}$. By Lemma 2.1, there is an ordinal number $\alpha<\kappa$ such that the set $x_{\alpha}$ omits infinitely many intervals $[a(n), a(n+1))$. Thus, the set

$$
b:=\left\{n \in \mathbb{N}: x_{\alpha} \cap[a(n), a(n+1))=\emptyset\right\}
$$

is an element of $[\mathbb{N}]^{\infty}$ and

$$
x_{\alpha} \cap \bigcup_{n \in b}[a(n), a(n+1))=\emptyset .
$$

For each ordinal number $\beta<\kappa$ with $\alpha<\beta$, we have $x_{\alpha}{ }^{*} \supseteq x_{\beta}$, and thus

$$
x_{\beta} \cap \bigcup_{n \in b}[a(n), a(n+1)) \in \text { Fin. }
$$

This observation motivates the following definition. 
Definition 2.2. Let $\kappa$ be an uncountable ordinal number. A set $X \subseteq[\mathbb{N}]^{\infty}$ with $|X| \geq \kappa$ is a $\kappa$-generalized tower if for each function $a \in[\mathbb{N}]^{\infty}$, there are sets $b \in[\mathbb{N}]^{\infty}$ and $S \subseteq X$ with $|S|<\kappa$ such that

$$
x \cap \bigcup_{n \in b}[a(n), a(n+1)) \in \text { Fin }
$$

for all sets $x \in X \backslash S$.

Let $\kappa$ be an uncountable ordinal number. Every $\kappa$-unbounded tower in $[\mathbb{N}]^{\infty}$ is a $\kappa$ generalized tower. The forthcoming Lemma 2.3 shows that the notion of $\kappa$-unbounded tower may capture wider class of sets than $\kappa$-unbounded towers.

A set $B \subseteq[\mathbb{N}]^{\infty}$ is groupwise dense if:

(1) for all sets $y \in[\mathbb{N}]^{\infty}$ and $b \in B$, if $y \subseteq^{*} b$, then $y \in B$,

(2) for each function $b \in[\mathbb{N}]^{\infty}$, there is a set $c \in[\mathbb{N}]^{\infty}$ such that $\bigcup_{n \in c}[b(n), b(n+1)) \in B$.

The groupwise density number $\mathfrak{g}$ is the minimal cardinality of a family of groupwise dense sets in $[\mathbb{N}]^{\infty}$ with empty intersection. For function $f, g \in \mathbb{N}^{\mathbb{N}}$, let $f \circ g \in \mathbb{N}^{\mathbb{N}}$ be a function such that $(f \circ g)(n):=f(g(n))$ for all natural numbers $n$.

Lemma 2.3. Let $\kappa$ be an uncountable ordinal number and $\lambda$ be an ordinal number with $\lambda<\mathfrak{g}$. Let $X:=\bigcup_{\alpha<\lambda} X_{\alpha}$ be a union of $\kappa$-generalized towers.

(1) If $\lambda<\mathrm{cf}(\kappa)$, then $X$ is a $\kappa$-generalized tower.

(2) If $\lambda \geq \operatorname{cf}(\kappa)$, then $X$ is a $(\kappa \cdot \lambda)^{+}$-generalized tower.

Proof. (1) Fix a function $a \in[\mathbb{N}]^{\infty}$ and an ordinal number $\alpha<\lambda$. Let $B_{\alpha}$ be the set of all sets $b \in[\mathbb{N}]^{\infty}$ such that

$$
x \cap \bigcup_{n \in b}[a(n), a(n+1)) \in \text { Fin, }
$$

for all but less than $\kappa$ sets $x \in X_{\alpha}$. The set $B_{\alpha}$ is groupwise dense: Fix a set $c \in[\mathbb{N}]^{\infty}$. There is a set $b \in[\mathbb{N}]^{\infty}$ such that

$$
x \cap \bigcup_{n \in b}[(a \circ c)(n),(a \circ c)(n+1)) \in \text { Fin, }
$$

for all but less than $\kappa$ sets $x \in X$. Let

$$
d:=\bigcup_{n \in b}[c(n), c(n+1))
$$

Fix a natural number $i \in d$. There is a natural number $n \in b$ with $i \in[c(n), c(n+1))$. Then $[a(i), a(i+1)) \subseteq[a(c(n)), a(c(n+1)))$. Thus,

$$
\bigcup_{i \in d}[a(i), a(i+1)) \subseteq \bigcup_{n \in b}[a(c(n)), a(c(n+1)))=\bigcup_{n \in b}[(a \circ c)(n),(a \circ c)(n+1)) .
$$

It follows that $d \in B_{\alpha}$.

Since $\lambda<\mathfrak{g}$, there is a set $b \in \bigcap_{\alpha<\lambda} B_{\alpha}$. For each ordinal number $\alpha<\lambda$, there is a set $S_{\alpha} \subseteq X_{\alpha}$ with $\left|S_{\alpha}\right|<\kappa$ such that condition (2.3.1) holds for all sets $x \in X_{\alpha} \backslash S_{\alpha}$. Then condition (2.3.1) holds for all sets $x \in X \backslash \bigcup_{\alpha<\lambda} S_{\alpha}$. Since $\left|\bigcup_{\alpha<\lambda} S_{\alpha}\right|<\kappa$, the set $X$ is a $\kappa$-generalized tower.

(2) Proceed as in (1) with the exception that $\left|\bigcup_{\alpha<\lambda} S_{\alpha}\right|<(\kappa \cdot \lambda)^{+}$. 


\section{Products of Sets Satisfying $\mathrm{S}_{1}(\Gamma, \Gamma)$}

For a class $\mathcal{A}$ of covers of spaces and a space $X$, let $\mathcal{A}(X)$ be the family of all covers of $X$ from the class $\mathcal{A}$. Let $\mathcal{A}, \mathcal{B}$ be classes of covers of spaces. A space $X$ satisfies $\mathrm{S}_{1}(\mathcal{A}, \mathcal{B})$ if for each sequence $\mathcal{U}_{1}, \mathcal{U}_{2}, \ldots \in \mathcal{A}(X)$, there are sets $U_{1} \in \mathcal{U}_{1}, U_{2} \in \mathcal{U}_{2}, \ldots$ such that $\left\{U_{n}: n \in \mathbb{N}\right\} \in \mathcal{B}(X)$. A Borel cover of a space is a cover whose members are Borel subsets of the space. Let $\Gamma_{\text {Bor }}$ be the class of all Borel $\gamma$-covers of spaces. For a property of spaces $\mathbf{P}$, let non $(\mathbf{P})$ be the minimal cardinality of a set of reals with no property $\mathbf{P}$. We have [12, Theorem 4.7], [26, Theorem 27(2)]

$$
\operatorname{non}\left(\mathrm{S}_{1}\left(\Gamma_{\text {Bor }}, \Gamma_{\text {Bor }}\right)\right)=\operatorname{non}\left(\mathrm{S}_{1}(\Gamma, \Gamma)\right)=\mathfrak{b} .
$$

In the Laver model, all sets of reals satisfying $S_{1}(\Gamma, \Gamma)$ have cardinality strictly smaller than $\mathfrak{b}$ [15, Theorem 3.6], and thus they are trivial; it implies that the properties $S_{1}\left(\Gamma_{\text {Bor }}, \Gamma_{\text {Bor }}\right)$ and $\mathrm{S}_{1}(\Gamma, \Gamma)$ are equivalent. In order to construct nontrivial examples of sets of reals with the above properties, additional set-theoretic assumptions are needed. A set of reals of cardinality at least $\mathfrak{b}$ is a $\mathfrak{b}$-Sierpiński set, if its intersection with every Lebesgue-null set has cardinality less than $\mathfrak{b}$. Every $\mathfrak{b}$-Sierpiński set satisfies $S_{1}\left(\Gamma_{\text {Bor }}, \Gamma_{\text {Bor }}\right)$ and assuming that $\operatorname{cov}(\mathcal{N})=$ $\operatorname{cof}(\mathcal{N})=\mathfrak{b}$, such a set exists (definitions of cardinal characteristics of the continuum and relations between them can be found in the Blass survey [5]). For functions $a, b \in[\mathbb{N}]^{\infty}$, we write $a \leq^{*} b$ if the set $\{n: b(n)<a(n)\}$ is finite. A set $A \subseteq[\mathbb{N}]^{\infty}$ is bounded if there is a function $b \in[\mathbb{N}]^{\infty}$ such that $a \leq^{*} b$ for all functions $a \in A$, denoted $A \leq^{*} b$. A subset of $[\mathbb{N}]^{\infty}$ is dominating if for every function $a \in[\mathbb{N}]^{\infty}$, there is a function $b$ from the set such that $a \leq^{*} b$. Let $\mathfrak{d}$ be the minimal cardinality of a dominating set in $[\mathbb{N}]^{\infty}$. Assuming $\mathfrak{p}=\mathfrak{b}$ or $\mathfrak{b}<\mathfrak{d}$, a $\mathfrak{b}$-unbounded tower exists [15, Lemma 2.2]. Also if for an infinite ordinal number $\kappa$, there is a $\kappa$-generalized tower, then there is a $\mathfrak{b}$-generalized tower [15, Proposition 2.4]. Each $\mathfrak{b}$-unbounded tower set satisfies $S_{1}(\Gamma, \Gamma)$ ([25, Theorem 6], [15, Proposition 2.5]), but not $S_{1}\left(\Gamma_{\text {Bor }}, \Gamma_{\text {Bor }}\right)$ [15, Corollary 2.10].

A subfamily of $[\mathbb{N}]^{\infty}$ is open if it is closed under almost subsets, and it is dense if each set from $[\mathbb{N}]^{\infty}$ has an almost subset in the family. The density number $\mathfrak{h}$ is the minimal cardinality of a collection of open dense families in $[\mathbb{N}]^{\infty}$ with empty intersection. Let add $\left(S_{1}(\Gamma, \Gamma)\right)$ be the minimal cardinality of a family of sets of reals satisfying $S_{1}(\Gamma, \Gamma)$ whose union does not satisfy $\mathrm{S}_{1}(\Gamma, \Gamma)$. By the result of Scheepers [24, Theorem 5] we have add $\left(\mathrm{S}_{1}(\Gamma, \Gamma)\right) \geq \mathfrak{h}$. Miller and Tsaban proved that, assuming add $\left(S_{1}(\Gamma, \Gamma)\right)=\mathfrak{b}$, each $\mathfrak{b}$-unbounded tower set satisfies $\mathrm{S}_{1}(\Gamma, \Gamma)$ in all finite powers. By the result of Miller, Tsaban and Zdomskyy, the product space of a $\mathfrak{b}$-unbounded tower set and a set satisfying $S_{1}\left(\Gamma_{\text {Bor }}, \Gamma_{\text {Bor }}\right)$, satisfies $S_{1}(\Gamma, \Gamma)[16$, Theorem 7.1]. In the forthcoming Theorem 3.1, we generalize the above results. In the proof we develop methods of Miller, Tsaban and Zdomskyy [16, Theorem 7.1] and combine them with tools invented by Tsaban and the first named author [27, Lemma 5.1].

Theorem 3.1. The product space of finitely many $\mathfrak{b}$-generalized tower sets and a set satisfying $\mathrm{S}_{1}\left(\Gamma_{\text {Bor }}, \Gamma_{\text {Bor }}\right)$, satisfies $\mathrm{S}_{1}(\Gamma, \Gamma)$.

In order to prove Theorem $\underline{3.1}$, we need the following notions and auxiliary results. Let $\overline{\mathbb{N}}$ be the set $\mathbb{N} \cup\{\infty\}$ with the discrete topology and $n<\infty$ for all natural numbers $n$. In the space $\overline{\mathbb{N}}^{\mathbb{N}}$, define relation $\leq^{*}$, analogously as in $[\mathbb{N}]^{\infty}$. For a set $x \in \mathrm{P}(\mathbb{N})$, let $x^{\mathrm{c}}:=\mathbb{N} \backslash x$.

Lemma 3.2. Let $m$ be a natural number, $Y$ be a set satisfying $\mathrm{S}_{1}\left(\Gamma_{\mathrm{Bor}}, \Gamma_{\mathrm{Bor}}\right)$ and $\left\{U_{n}: n \in\right.$ $\mathbb{N}\} \in \Gamma\left(\operatorname{Fin}^{m} \times Y\right)$ be a family of open sets in $\mathrm{P}(\mathbb{N})^{m} \times Y$. 
(1) There are Borel functions $f, g: Y \rightarrow \overline{\mathbb{N}}^{\mathbb{N}}$ such that for all points $x \in\left([\mathbb{N}]^{\infty}\right)^{m}$ and $y \in Y$, and all natural numbers $n$ :

If $x_{i} \cap[f(y)(n), g(y)(n))=\emptyset$ for all natural numbers $i \leq m$, then $(x, y) \in U_{n}$.

(2) There is an increasing function $c \in \mathbb{N}^{\mathbb{N}}$ such that for each point $y \in Y$, we have

$$
c(n) \leq f(y)(c(n+1)) \leq g(y)(c(n+1))<c(n+2),
$$

for all but finitely many natural numbers $n$.

Proof. (1) Define a function $d: Y \rightarrow \mathbb{N}^{\mathbb{N}}$ as follows: Fix a point $y \in Y$. Let $d(y)(1)$ be the minimal natural number such that

$$
\{\emptyset\}^{m} \times\{y\} \subseteq \bigcap_{k \geq d(y)(1)} U_{k}
$$

and, for each natural number $i$, let $d(y)(i+1)$ be the minimal natural number greater than $d(y)(i)$ such that

$$
\mathrm{P}([1, i+1))^{m} \times\{y\} \subseteq \bigcap_{k \geq d(y)(i+1)} U_{k}
$$

The function $d$ is Borel: We have

$$
\{y \in Y: d(y)(1)=1\}=\left\{y \in Y:\{\emptyset\}^{m} \times\{y\} \subseteq \bigcap_{k \geq 1} U_{k}\right\},
$$

and

$$
\{y \in Y: d(y)(1)=j+1\}=\left\{y \in Y:\{\emptyset\}^{m} \times\{y\} \subseteq \bigcap_{k \geq j+1} U_{k} \backslash U_{j}\right\},
$$

for all natural numbers $j$; the above sets are Borel. Fix natural numbers $i, j$, and assume that the set

$$
\{y \in Y: d(y)(i)=j\}
$$

is Borel. Fix a point $y \in Y$ such that $d(y)(i+1)=j+1$. We have

$$
\mathrm{P}([1, i+1))^{m} \times\{y\} \subseteq \bigcap_{k \geq j+1} U_{k} .
$$

If $\mathrm{P}([1, i+1))^{m} \times\{y\} \subseteq U_{j}$, then $d(y)(i)=j$, otherwise there is an element $t \in \mathrm{P}([1, i+1))^{m}$ with $(t, y) \notin U_{j}$. Thus,

$$
\begin{aligned}
\{y \in Y: d(y)(i+1)=j+1\}= & \bigcap_{t \in \mathrm{P}([1, i+1))^{m}}\left\{y \in Y:(t, y) \in \bigcap_{k \geq j+1} U_{k}\right\} \cap \\
& \left(\{y \in Y: d(y)(i)=j\} \cup \bigcup_{t \in \mathrm{P}([1, i+1))^{m}}\left\{y \in Y:(t, y) \notin U_{j}\right\}\right) .
\end{aligned}
$$

Then the set $\{y \in Y: d(y)(i+1)=j+1\}$ is Borel.

For a point $y \in Y$, let $f(y) \in \overline{\mathbb{N}}^{\mathbb{N}}$ be a function such that:

$$
f(y)(n):= \begin{cases}\infty, & \text { for } n \in[1, d(y)(1)), \\ i, & \text { for } n \in[d(y)(i), d(y)(i+1)), i \in \mathbb{N} .\end{cases}
$$

For natural numbers $i, n$, we have

$$
\{y \in Y: f(y)(n)=\infty\}=\{y \in Y: n<d(y)(1)\}
$$


and

$$
\{y \in Y: f(y)(n)=i\}=\{y \in Y: d(y)(i) \leq n<d(y)(i+1)\} .
$$

Since the function $d$ is Borel, the function $f: Y \rightarrow \overline{\mathbb{N}}^{\mathbb{N}}$, defined above, is Borel as well.

For each natural number $n$, let $U_{1}^{(n)}, U_{2}^{(n)}, \ldots$ be an increasing sequence of clopen sets in $\mathrm{P}(\mathbb{N})^{m} \times Y$ such that $U_{n}=\bigcup_{k} U_{k}^{(n)}$. Fix a point $y \in Y$. Define a function $g(y) \in \overline{\mathbb{N}}^{\mathbb{N}}$ in the following way: Let $n$ be a natural number. If $f(y)(n)=\infty$, then $g(y)(n):=\infty$. Assume that $f(y)(n) \in \mathbb{N}$. There is a natural number $j>f(y)(n)$ such that

$$
\mathrm{P}\left([f(y)(n), j)^{\mathrm{c}}\right)^{m} \times\{y\} \subseteq U_{j}^{(n)}:
$$

Let $k$ be the minimal natural number with

$$
\mathrm{P}([1, f(y)(n)))^{m} \times\{y\} \subseteq U_{k}^{(n)} .
$$

Let $j$ be the minimal natural number with $j>f(y)(n)$ and

$$
\mathrm{P}\left([f(y)(n), j)^{\mathrm{c}}\right)^{m} \times\{y\} \subseteq U_{k}^{(n)} .
$$

Since the sets $U_{1}^{(n)}, U_{2}^{(n)}, \ldots$ are ascending, we have

$$
\mathrm{P}\left([f(y)(n), j)^{\mathrm{c}}\right)^{m} \times\{y\} \subseteq U_{j}^{(n)} .
$$

Let $g(y)(n)>f(y)(n)$ be the minimal natural number with

$$
\mathrm{P}\left([f(y)(n), g(y)(n))^{c}\right)^{m} \times\{y\} \subseteq U_{g(y)(n)}^{(n)} .
$$

The set $\mathrm{P}\left([f(y)(n), g(y)(n))^{\mathrm{c}}\right)^{m}$ is compact, and thus there is an open set $V_{n}^{y}$ in $Y$ such that

$$
\mathrm{P}\left([f(y)(n), g(y)(n))^{\mathrm{c}}\right)^{m} \times V_{n}^{y} \subseteq U_{g(y)(n)}^{(n)} .
$$

The function $g$ is Borel: Fix a natural number $n$. We have $\{y \in Y: g(y)(n)=\infty\}=$ $\{y \in Y: f(y)(n)=\infty\}$. Since the function $f$ is Borel, the latter set is Borel. For a natural number $k$, we have

$$
\{y \in Y: g(y)(n)=k\}=\bigcup_{i<k}\{y \in Y: f(y)(n)=i \wedge g(y)(n)=k\} .
$$

In order to show that such a set is Borel, it is enough to show that for natural numbers $i, k$ with $i<k$, the set $\{y \in Y: f(y)(n)=i \wedge g(y)(n)=k\}$ is Borel: Fix natural numbers $i, k$ with $i<k$. Let $y \in Y$ be a point such that $f(y)(n)=i$ and $g(y)(n)=k$. For each point $y^{\prime} \in V_{n}^{y}$ such that $f\left(y^{\prime}\right)(n)=i$, we have

$$
\mathrm{P}\left(\left[f\left(y^{\prime}\right)(n), g(y)(n)^{\mathrm{c}}\right)^{m} \times\left\{y^{\prime}\right\} \subseteq \mathrm{P}\left([f(y)(n), g(y)(n))^{\mathrm{c}}\right)^{m} \times V_{n}^{y} \subseteq U_{g(y)(n)}^{(n)} .\right.
$$

By the minimality of the number $g\left(y^{\prime}\right)(n)$, we have

$$
g\left(y^{\prime}\right)(n) \leq g(y)(n)
$$

If $k=i+1$, then $g\left(y^{\prime}\right)(n)=g(y)(n)$, and thus the set $\{y \in Y: f(y)(n)=i \wedge g(y)(n)=k\}$ is Borel (in fact, it is even open). Now assume that for a natural number $k>i+1$, the set $\{y \in Y: f(y)(n)=i \wedge g(y)(n)<k\}$ is Borel. By the above inequality (3.2.1), we have

$$
\begin{gathered}
\{y \in Y: f(y)(n)=i \wedge g(y)(n)=k\}= \\
\left(\{y \in Y: f(y)(n)=i\} \cap \bigcup\left\{V_{n}^{y}: g(y)(n)=k\right\}\right) \backslash\{y \in Y: f(y)(n)=i \wedge g(y)(n)<k\} .
\end{gathered}
$$

Since the function $f$ is Borel, the set $\{y \in Y: f(y)(n)=i \wedge g(y)(n)=k\}$ is Borel. 
(2) Let $d$ and $g$ be the functions from the proof of (1). For each point $y \in Y$, we have $g(y)(n)=\infty$ for finitely many natural numbers $n$. Since the functions $d$ and $g$ are Borel and the set $Y$ satisfies $\mathrm{S}_{1}\left(\Gamma_{\text {Bor }}, \Gamma_{\text {Bor }}\right)$, there is an increasing function $b \in \mathbb{N}^{\mathbb{N}}$ such that $\{d(y), g(y): y \in Y\} \leq^{*} b[26$, Theorem 1]. We may assume that $b(1) \neq 1$. Let $c(1):=b(1)$ and $c(n+1):=b(c(n))$ for all natural numbers $n$. For each point $y \in Y$, we have

$$
c(n) \leq f(y)(c(n+1)) \leq g(y)(c(n+1))<c(n+2)
$$

for all but finitely many natural numbers $n$ : Fix a point $y \in Y$. There is a natural number $l$ such that

$$
d(y)(n) \leq b(n) \text { and } g(y)(n) \leq b(n),
$$

for all natural numbers $n \geq l$. Let $n$ be a natural number with $n \geq l$. Since

$$
d(y)(c(n)) \leq b(c(n))=c(n+1)
$$

and the function $f(y)$ is nondecreasing for arguments greater than or equal to $d(y)(1)$, we have

$$
c(n)=f(y)(d(y)(c(n))) \leq f(y)(c(n+1)) \leq g(y)(c(n+1)) \leq b(c(n+1))=c(n+2) .
$$

Lemma 3.3. Let $X$ be a space such that for every sequence $\mathcal{U}_{1}, \mathcal{U}_{2}, \ldots \in \Gamma(X)$, there are sets $U_{1} \in \mathcal{U}_{1}, U_{2} \in \mathcal{U}_{2}, \ldots$ and a space $X^{\prime} \subseteq X$ satisfying $S_{1}(\Gamma, \Gamma)$ such that $\left\{U_{n}: n \in \mathbb{N}\right\} \in$ $\Gamma\left(X \backslash X^{\prime}\right)$. Then the space $X$ satisfies $\mathrm{S}_{1}(\Gamma, \Gamma)$.

Proof. Let $\mathcal{U}_{n}=\left\{U_{m}^{(n)}: m \in \mathbb{N}\right\}$ for all natural numbers $n$. We may assume that for all natural numbers $n, m$, we have

$$
U_{m}^{(n+1)} \subseteq U_{m}^{(n)} .
$$

For each natural number $n$, we have $\left\{U_{m}^{(n)}: m \geq n\right\} \in \Gamma(X)$. Then there are a function $f \in \mathbb{N}^{\mathbb{N}}$ and a space $X^{\prime} \subseteq X$ such that $\left\{U_{f(n)}^{(n)}: n \in \mathbb{N}\right\} \in \Gamma\left(X \backslash X^{\prime}\right)$. The range of the function $f$ is infinite, and thus there is a function $d \in[\mathbb{N}]^{\infty}$ such that $(f \circ d) \in[\mathbb{N}]^{\infty}$. Let $f^{\prime}:=f \circ d$. Since $U_{f^{\prime}(n)}^{(d(n))} \subseteq U_{f^{\prime}(n)}^{(n)}$, we have $\left\{U_{f^{\prime}(n)}^{(n)}: n \in \mathbb{N}\right\} \in \Gamma\left(X \backslash X^{\prime}\right)$.

Since the space $X^{\prime}$ satisfies $S_{1}(\Gamma, \Gamma)$, the set

$$
\left\{a \in[\mathbb{N}]^{\infty}:\left\{U_{a(n)}^{(n)}: n \in \mathbb{N}\right\} \in \Gamma\left(X^{\prime}\right)\right\}
$$

is open and dense [24, Theorem 5]. Thus, there is a function $a \in[\mathbb{N}]^{\infty}$ with $a \subseteq f^{\prime}$ such that $\left\{U_{a(n)}^{(n)}: n \in \mathbb{N}\right\} \in \Gamma\left(X^{\prime}\right)$. There is a function $c \in[\mathbb{N}]^{\infty}$ such that $a=f^{\prime} \circ c$. Then $\left\{U_{a(n)}^{(c(n))}: n \in \mathbb{N}\right\} \in \Gamma\left(X \backslash X^{\prime}\right)$. Since $U_{a(n)}^{(c(n))} \subseteq U_{a(n)}^{(n)}$, we have $\left\{U_{a(n)}^{(n)}: n \in \mathbb{N}\right\} \in \Gamma(X)$.

Proof of Theorem 3.1. Let $Y$ be a set satisfying $\mathrm{S}_{1}\left(\Gamma_{\text {Bor }}, \Gamma_{\text {Bor }}\right)$.

Let $X$ be a $\mathfrak{b}$-generalized tower and $\mathcal{U}_{1}, \mathcal{U}_{2}, \ldots \in \Gamma((X \cup$ Fin $) \times Y)$ be a sequence of families of open sets in $\mathrm{P}(\mathbb{N}) \times Y$ such that $\mathcal{U}_{k}=\left\{U_{n}^{(k)}: n \in \mathbb{N}\right\}$ for all natural numbers $k$. For each natural number $k$, let $f_{k}, g_{k}, c_{k}$ be functions from Lemma 3.2 applied to the family $\mathcal{U}_{k}$. Let $h: Y \rightarrow \mathbb{N}^{\mathbb{N}}$ be a function such that $h(y)(k)$ is the minimal natural number $n$ such that for all natural numbers $j$ with $j \geq n$, we have

$$
c_{k}(j) \leq f_{k}(y)\left(c_{k}(j+1)\right)<g_{k}(y)\left(c_{k}(j+1)\right)<c_{k}(j+2) .
$$

Since the functions $f_{k}$ and $g_{k}$ are Borel, so is the function $h$, and hence there is an increasing function $z \in \mathbb{N}^{\mathbb{N}}$ such that $\{h(y): y \in Y\} \leq^{*} z$. We may assume that

$$
c_{k+1}(z(k+1))>c_{k}(z(k)+2)
$$


for all natural numbers $k$. Since the set $X$ is a $\mathfrak{b}$-generalized tower, there are a set $b \in[\mathbb{N}]^{\infty}$ and a set $S \subseteq X$ with $|S|<\mathfrak{b}$ such that

$$
x \cap \bigcup_{k \in b}\left[c_{k}(z(k)), c_{k}(z(k)+2)\right) \in \text { Fin }
$$

for all sets $x \in X \backslash S$.

We have $\left\{U_{c_{k}(z(k+1))}^{(k)}: k \in \mathbb{N}\right\} \in \Gamma((X \backslash S) \times Y)$ : Fix points $x \in X \backslash S$ and $y \in Y$. There is a natural number $l$ such that for all natural numbers $k \geq l$, we have

$$
\begin{gathered}
c_{k}(z(k)) \leq f_{k}(y)\left(c_{k}(z(k)+1)\right)<g_{k}(y)\left(c_{k}(z(k)+1)\right)<c_{k}(z(k)+2), \\
x \cap\left[c_{k}(z(k)), c_{k}(z(k)+2)\right)=\emptyset,
\end{gathered}
$$

and thus

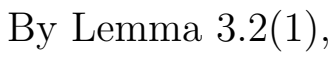

$$
x \cap\left[f_{k}(y)(z(k+1)), g_{k}(y)(z(k+1))\right)=\emptyset .
$$

$$
(x, y) \in \bigcap_{k \geq l} U_{z(k+1)}^{(k)} .
$$

Since $|S|<\mathfrak{b}$ and $\operatorname{add}\left(\mathrm{S}_{1}\left(\Gamma_{\text {Bor }}, \Gamma_{\text {Bor }}\right)\right)=\mathfrak{b}[28$, Corollary 2.4], the product space $(S \cup$ Fin $) \times Y$ satisfies $\mathrm{S}_{1}\left(\Gamma_{\text {Bor }}, \Gamma_{\text {Bor }}\right)$. By Lemma 3.3, the product space $(X \cup$ Fin $) \times Y$ satisfies $\mathrm{S}_{1}(\Gamma, \Gamma)$.

Let $m$ be a natural number with $m>1$ and assume that the statement is true for $m-1$ $\mathfrak{b}$-generalized tower sets. Let $X_{1}, \ldots, X_{m}$ be $\mathfrak{b}$-generalized towers in $[\mathbb{N}]^{\infty}$ and

$$
\mathcal{U}_{1}, \mathcal{U}_{2}, \ldots \in \Gamma\left(\left(X_{1} \cup \text { Fin }\right) \times \cdots \times\left(X_{m} \cup \text { Fin }\right) \times Y\right)
$$

be a sequence of families of open sets in $\mathrm{P}(\mathbb{N})^{m} \times Y$ such that $\mathcal{U}_{k}=\left\{U_{n}^{(k)}: n \in \mathbb{N}\right\}$ for all natural numbers $k$. We proceed analogously to the previous case. For each natural number $k$, let $f_{k}, g_{k}, c_{k}$ be functions from Lemma 3.2 applied to the family $\mathcal{U}_{k}$. Let $h: Y \rightarrow \mathbb{N}^{\mathbb{N}}$ be a function such that $h(y)(k)$ is the minimal natural number $n$ such that for all natural numbers $j$ with $j \geq n$, we have

$$
c_{k}(j) \leq f_{k}(y)\left(c_{k}(j+1)\right)<g_{k}(y)\left(c_{k}(j+1)\right)<c_{k}(j+2) .
$$

Since the functions $f_{k}$ and $g_{k}$ are Borel, so is the function $h$, and hence there is an increasing function $z \in \mathbb{N}^{\mathbb{N}}$ such that $\{h(y): y \in Y\} \leq^{*} z$. We may assume that

$$
c_{k+1}(z(k+1))>c_{k}(z(k)+2)
$$

for all natural numbers $k$. By Lemma 2.3(1), the set $X:=\bigcup_{i \leq m} X_{i}$ is a $\mathfrak{b}$-generalized tower. Then there are a set $b \in[\mathbb{N}]^{\infty}$ and a set $S \subseteq X$ with $|S|<\mathfrak{b}$ such that

$$
x \cap \bigcup_{k \in b}\left[c_{k}(z(k)), c_{k}(z(k)+2)\right) \in \text { Fin }
$$

for all sets $x \in X \backslash S$.

We have $\left\{U_{c_{k}(z(k+1))}^{(k)}: k \in \mathbb{N}\right\} \in \Gamma\left((X \backslash S)^{m} \times Y\right)$ : Fix points $x \in(X \backslash S)^{m}$ and $y \in Y$. There is a natural number $l$ such that for all natural numbers $k \geq l$, we have

$$
\begin{gathered}
c_{k}(z(k)) \leq f_{k}(y)\left(c_{k}(z(k)+1)\right)<g_{k}(y)\left(c_{k}(z(k)+1)\right)<c_{k}(z(k)+2), \\
x_{i} \cap\left[c_{k}(z(k)), c_{k}(z(k)+2)\right)=\emptyset \text { for all natural numbers } i \leq m,
\end{gathered}
$$

and thus

$$
x_{i} \cap\left[f_{k}(y)(z(k+1)), g_{k}(y)(z(k+1))\right)=\emptyset \text { for all natural numbers } i \leq m .
$$


By Lemma $3.2(1)$,

$$
(x, y) \in \bigcap_{k \geq l} U_{z(k+1)}^{(k)} .
$$

Since $|S|<\mathfrak{b}$ and $\operatorname{add}\left(\mathrm{S}_{1}\left(\Gamma_{\text {Bor }}, \Gamma_{\text {Bor }}\right)\right)=\mathfrak{b}[28$, Corollary 2.4$]$, the product space $\left(\left(X_{j} \cap S\right) \cup\right.$ Fin $) \times Y$ satisfies $\mathrm{S}_{1}\left(\Gamma_{\text {Bor }}, \Gamma_{\text {Bor }}\right)$ for all natural numbers $j \leq m+1$, and the set

$$
\bigcup_{j \leq m} \prod_{\substack{i \leq m \\ i \neq j}}\left(X_{i} \cup \text { Fin }\right) \times\left(\left(X_{j} \cap S\right) \cup \text { Fin }\right) \times Y
$$

satisfies $\mathrm{S}_{1}(\Gamma, \Gamma)$. By Lemma 3.3, the product space $\left(X_{1} \cup\right.$ Fin $) \times \cdots \times\left(X_{m} \cup\right.$ Fin $) \times Y$ satisfies $\mathrm{S}_{1}(\Gamma, \Gamma)$.

Corollary 3.4. Each $\mathfrak{b}$-generalized tower set satisfies $\mathrm{S}_{1}(\Gamma, \Gamma)$ in all finite powers.

The properties $S_{1}(\Gamma, \Gamma)$ and $S_{1}\left(\Gamma_{\text {Bor }}, \Gamma_{\text {Bor }}\right)$ are closely related to local properties of functions spaces. Let $X$ be a space and $\mathrm{C}_{\mathrm{p}}(X)$ be the set of all continuous real-valued functions on $X$ with the pointwise convergence topology. A sequence $f_{1}, f_{2}, \ldots \in \mathrm{C}_{\mathrm{p}}(X)$ converges quasinormally to the constant zero function $\mathbf{0}$, if there is a sequence of positive real numbers $\epsilon_{1}, \epsilon_{2}, \ldots$ converging to zero such that for any point $x \in X$, we have $\left|f_{n}(x)\right|<\epsilon_{n}$ for all but finitely many natural numbers $n$. A space $X$ is a $Q N$-space ( $w Q N$-space) if every sequence $f_{1}, f_{2}, \ldots \in \mathrm{C}_{\mathrm{p}}(X)$ converging pointwise to $\mathbf{0}$, converges (has a subsequence converging) quasinormally to 0. By a breakthrough result of Tsaban and Zdomskyy [31, Theorem 2], a space is a QN-space if and only if all Borel images of the space in $\mathbb{N}^{\mathbb{N}}$ are bounded [26. Theorem 1]. In consequence, a space satisfies $S_{1}\left(\Gamma_{\text {Bor }}, \Gamma_{\text {Bor }}\right)$ if and only if it is a QN-space. Every perfectly normal space satisfying $S_{1}(\Gamma, \Gamma)$, is a wQN-space [7, Theorem 7]. QN-spaces, wQN-spaces and their variations were extensively studied by Bukovský, Haleš, Recław, Sakai and Scheepers [6, 7, 8, 11, 20, 21, 24]. We have the following corollary from Theorem 3.1.

Corollary 3.5. The product space of finitely many $\mathfrak{b}$-generalized tower sets and a $Q N$-space, is a $w Q N$-space.

These results can also be formulated as dealing with Arhangel'skiı̀'s properties $\alpha_{i}$ for spaces of continuous real-valued functions [25, 1, 2, .

\section{Generalized towers And productivity of $\left(\begin{array}{l}\Omega \\ \Gamma\end{array}\right)$}

A space is Fréchet-Urysohn if each point in the closure of a set is a limit of a sequence from the set. By the celebrating result of Gerlits and Nagy, for a set of reals $X$, the space $\mathrm{C}_{\mathrm{p}}(X)$ is Fréchet-Urysohn if and only if the set $X$ satisfies $\left(\begin{array}{l}\Omega \\ \Gamma\end{array}\right)\left[9\right.$, Theorem 2]. The property $\left(\begin{array}{r}\Omega \\ \Gamma\end{array}\right)$ is productive if each space satisfying $\left(\frac{\Omega}{\Gamma}\right)$, is productively $\left(\frac{\Omega}{\Gamma}\right)$. The property $\left(\frac{\Omega}{\Gamma}\right)$ is preserved by finite powers, but productivity of $\left(\begin{array}{l}\Omega \\ \Gamma\end{array}\right)$ is independent of ZFC. In the Laver model, all sets of reals satisfying $(\underset{\Gamma}{\Omega})$ are countable ([9, Theorem 2], [23, Theorem 17], [13]), and thus $\left(\frac{\Omega}{\Gamma}\right)$ is productive; if the Continuum Hypothesis holds, then $\left(\begin{array}{c}\Omega \\ \Gamma\end{array}\right)$ is not productive [16, Theorem 3.2.]. Miller, Tsaban and Zdomskyy proved that, each $\omega_{1}$-unbounded tower set is productively $\left(\begin{array}{l}\Omega \\ \Gamma\end{array}\right)$ [16, Theorem 2.8.]. The main result of this section is the following Theorem.

\section{Theorem 4.1.}

(1) The product space of finitely many $\mathfrak{p}$-generalized tower sets, satisfies $\left(\begin{array}{l}\Omega \\ \Gamma\end{array}\right)$.

(2) Assume that there is a $\mathfrak{p}$-generalized tower in $[\mathbb{N}]^{\infty}$. The following assertions are equivalent: 
(a) Each set of reals of cardinality smaller than $\mathfrak{p}$ is productively $\left(\begin{array}{l}\Omega \\ \Gamma\end{array}\right)$.

(b) Each $\mathfrak{p}$-generalized tower set is productively $\left(\begin{array}{c}\Omega \\ \Gamma\end{array}\right)$.

Let $\Omega$ be the class of all $\omega$-covers of spaces.

Lemma 4.2 (Galvin-Miller[10, Lemma 1.2]). Let $\mathcal{U}$ be a family of open sets in $\mathrm{P}(\mathbb{N})$ such that $\mathcal{U} \in \Omega\left(\right.$ Fin). There are a function $a \in[\mathbb{N}]^{\infty}$ and sets $U_{1}, U_{2}, \ldots \in \mathcal{U}$ such that for each set $x \in[\mathbb{N}]^{\infty}$ and all natural numbers $n$ :

$$
\text { If } x \cap[a(n), a(n+1))=\emptyset \text {, then } x \in U_{n} .
$$

For spaces $X$ and $Y$, let $X \sqcup Y$ be the disjoint union of these spaces. The product space $X \times Y$ satisfies $\left(\begin{array}{c}\Omega \\ \Gamma\end{array}\right)$ if and only if $X \sqcup Y$ satisfies $\left(\begin{array}{c}\Omega \\ \Gamma\end{array}\right)$ [14, Proposition 2.3]. For functions $a, b \in[\mathbb{N}]^{\infty}$, we write $a \leq b$ if $a(n) \leq b(n)$ for all natural numbers $n$.

Lemma 4.3. Let $X \subseteq[\mathbb{N}]^{\infty}$ be a $\mathfrak{p}$-generalized tower and $Y$ be a set such that for every subset $S \subseteq X$ with $|S|<\mathfrak{p}$, the product space $S \times Y$ satisfies $\left(\begin{array}{c}\Omega \\ \Gamma\end{array}\right)$. Then the product space $(X \cup$ Fin $) \times Y$ satisfies $\left(\begin{array}{l}\Omega \\ \Gamma\end{array}\right)$.

Proof. Let $\mathcal{U} \in \Omega((X \cup$ Fin $) \sqcup Y)$ be a family of open sets in $\mathrm{P}(\mathbb{N}) \sqcup \mathrm{P}(\mathbb{N})$. Let $S_{1}:=$ Fin. Fix a natural number $k>1$, and assume that the set $S_{k-1} \subseteq X$ with Fin $\subseteq S_{k-1}$ and $\left|S_{k-1}\right|<\mathfrak{p}$ has been already defined. Since $\left|S_{k-1}\right|<\mathfrak{p}$, there is a subfamily $\overline{\mathcal{U}}^{\prime}$ of $\mathcal{U}$ with $\mathcal{U}^{\prime} \in \Gamma\left(S_{k-1} \sqcup Y\right)$. Apply Lemma 4.2 to the family $\mathcal{U}^{\prime}$. Then there are a function $a_{k} \in[\mathbb{N}]^{\infty}$ and sets $U_{1}^{(k)}, U_{2}^{(k)}, \ldots \in \mathcal{V}$ such that for each set $x \in[\mathbb{N}]^{\infty}$ and all natural numbers $n$ :

$$
\text { If } x \cap\left[a_{k}(n), a_{k}(n+1)\right)=\emptyset \text {, then } x \in U_{n}^{(k)} \text {. }
$$

Since the set $X$ is a $\mathfrak{p}$-generalized tower, there are a set $b_{k} \in[\mathbb{N}]^{\infty}$ and a set $S_{k} \subseteq X$ with $S_{k-1} \subseteq S_{k}$ and $\left|S_{k}\right|<\mathfrak{p}$ such that

$$
x \cap \bigcup_{n \in b_{k}}\left[a_{k}(n), a_{k}(n+1)\right) \in \text { Fin }
$$

for all sets $x \in X \backslash S_{k}$. Since $\mathcal{U}^{\prime} \in \Gamma(Y)$, we have

$$
\left\{U_{b_{k}(j)}^{(k)}: j \in \mathbb{N}\right\} \in \Gamma\left(\left(\left(X \backslash S_{k}\right) \cup S_{k-1}\right) \sqcup Y\right) .
$$

There is a function $a \in[\mathbb{N}]^{\infty}$ such that for each natural number $k$, we have

$$
\left|\left(a_{k} \circ b_{k}\right) \cap[a(n), a(n+1))\right| \geq 2,
$$

for all but finitely many natural numbers $n$. Since the set $X$ is a $\mathfrak{p}$-generalized tower, there are a set $b \in[\mathbb{N}]^{\infty}$ and a set $S \subseteq X$ with $|S|<\mathfrak{p}$ such that

$$
x \cap \bigcup_{n \in b}[a(n), a(n+1)) \in \text { Fin. }
$$

for all sets $x \in X \backslash S$. We may assume that $\bigcup_{k} S_{k} \subseteq S$. The sets

$$
b_{k}^{\prime}:=\left\{i \in b_{k}:\left[a_{k}(i), a_{k}(i+1)\right) \subseteq \bigcup_{n \in b}[a(n), a(n+1))\right\}
$$

are infinite for all natural numbers $k$. Thus,

$$
\left\{U_{b_{k}^{\prime}(j)}^{(k)}: j \in \mathbb{N}\right\} \in \Gamma\left(\left(\left(X \backslash S_{k}\right) \cup S_{k-1}\right) \sqcup Y\right) .
$$


Since the sequence of the sets $S_{k}$ is increasing, we have $X=\bigcup_{k}\left(X \backslash S_{k}\right) \cup S_{k-1}$ and each point of $X$ belongs to all but finitely many sets $\left(X \backslash S_{k}\right) \cup S_{k-1}$. For each point $x \in S$, define

$$
g_{x}(k):= \begin{cases}0, & \text { if } x \notin\left(X \backslash S_{k}\right) \cup S_{k-1}, \\ \min \left\{j: x \in \bigcap_{i \geq j} U_{b_{k}^{\prime}(i)}^{k}\right\}, & \text { if } x \in\left(X \backslash S_{k}\right) \cup S_{k-1} .\end{cases}
$$

Since $|S|<\mathfrak{p}$, there is a function $g \in \mathbb{N}^{\mathbb{N}}$ with $\left\{g_{x}: x \in S\right\} \leq^{*} g$ and

$$
a_{k}\left(b_{k}^{\prime}(g(k)+1)\right)<a_{k+1}\left(b_{k+1}^{\prime}(g(k+1))\right),
$$

for all natural numbers $k$. Let

$$
\mathcal{W}_{k}:=\left\{U_{b_{k}^{\prime}(j)}^{(k)}: j \geq g(k)\right\}
$$

for all natural numbers $k$. Then $\mathcal{W}_{1}, \mathcal{W}_{2}, \ldots \in \Gamma(Y)$. We may assume that families $\mathcal{W}_{k}$ are pairwise disjoint. Since the properties $\left(\begin{array}{c}\Omega \\ \Gamma\end{array}\right)$ and $S_{1}(\Omega, \Gamma)$ are equivalent [9, Theorem 2], the set $Y$ satisfies $\mathrm{S}_{1}(\Omega, \Gamma)$. Then there is a function $h \in \mathbb{N}^{\mathbb{N}}$ such that $g \leq h$ and

$$
\left\{U_{b_{k}^{\prime}(h(k))}^{(k)}: k \in \mathbb{N}\right\} \in \Gamma(S \sqcup Y) \text {. }
$$

Fix a set $x \in X \backslash S$. By (4.3.3), for each natural number $k$, we have

$$
\bigcup_{n \in b_{k}^{\prime}}\left[a_{k}(n), a_{k}(n+1)\right) \subseteq \bigcup_{n \in b}[a(n), a(n+1)) .
$$

By (4.3.2), (4.3.4) and the fact that $g \leq h$, the set $x$ omits all but finitely many intervals

$$
\left[a_{k}\left(b_{k}^{\prime}(h(k))\right), a_{k}\left(b_{k}^{\prime}(h(k))+1\right)\right) .
$$

By (4.3.1), we have

$$
\left\{U_{b_{k}^{\prime}(h(k))}^{(k)}: k \in \mathbb{N}\right\} \in \Gamma(X \backslash S)
$$

Thus,

$$
\left\{U_{b_{k}^{\prime}(h(k))}^{(k)}: k \in \mathbb{N}\right\} \in \Gamma((X \cup \text { Fin }) \sqcup Y) .
$$

Proof of Theorem 4.1. (1) We prove a formally stronger assertion that the product space of finitely many $\mathfrak{p}$-generalized tower sets and a set of cardinality less than $\mathfrak{p}$, satisfies $\left(\begin{array}{l}\Omega \\ \Gamma\end{array}\right)$. By (a) and Lemma 4.3, the product space of a $\mathfrak{p}$-generalized tower set and a set of cardinality smaller than $\mathfrak{p}$, satisfies $\left(\begin{array}{c}\Omega \\ \Gamma\end{array}\right)$. Fix a natural number $m>1$. Let $X_{1}, \ldots, X_{m}$ be $\mathfrak{p}$-generalized towers in $[\mathbb{N}]^{\infty}$ and $Y$ be a set with $|Y|<\mathfrak{p}$. Assume that the product space

$$
Z:=\left(X_{1} \cup \text { Fin }\right) \times \cdots \times\left(X_{m-1} \cup \text { Fin }\right) \times Y
$$

satisfies $\left(\begin{array}{c}\Omega \\ \Gamma\end{array}\right)$. Fix a set $S \subseteq X_{m}$ with $|S|<\mathfrak{p}$. Since $|S \times Y|<\mathfrak{p}$, by the inductive assumption, the product space

$$
S \times Z=\left(X_{1} \cup \text { Fin }\right) \times \cdots \times\left(X_{m-1} \cup \text { Fin }\right) \times(S \times Y)
$$

satisfies $\left(\begin{array}{l}\Omega \\ \Gamma\end{array}\right)$. By Lemma 4.3, the product space $\left(X_{1} \cup\right.$ Fin $) \times \cdots \times\left(X_{m} \cup\right.$ Fin $) \times Y$ satisfies $\left(\begin{array}{l}\Omega \\ \Gamma\end{array}\right)$.

$(2)(\Rightarrow)$ Apply Lemma 4.3.

$(\Leftarrow)$ Let $A \subseteq[\mathbb{N}]^{\infty}$ be a set with $|A|<\mathfrak{p}$ and $Y$ be a set satisfying $\left(\begin{array}{r}\Omega \\ \Gamma\end{array}\right)$. Since $|A|<\mathfrak{p}$, there is an element $b \in[\mathbb{N}]^{\infty}$ such that $A \leq^{*} b$. Let $X \subseteq[\mathbb{N}]^{\infty}$ be a p-generalized tower such that $x \subseteq b$ for all sets $x \in X$. Then the set $Z:=X \cup A$ is a p-generalized tower. We have

$$
A=Z \cap\left\{x \in[\mathbb{N}]^{\infty}: b \leq^{*} x\right\},
$$


and thus the set $A$ is an $F_{\sigma}$ subset of $Z$. The property $\left(\begin{array}{l}\Omega \\ \Gamma\end{array}\right)$ is preserved by taking $F_{\sigma}$ subsets [10, Theorem 3]. Since the space $(Z \cup$ Fin $) \sqcup Y$ satisfies $\left(\begin{array}{c}\Omega \\ \Gamma\end{array}\right)$, the space $A \sqcup Y$ satisfies $\left(\begin{array}{l}\Omega \\ \Gamma\end{array}\right)$, too.

\section{Products of sets satisfying $\left(\begin{array}{l}\Omega \\ \Gamma\end{array}\right)$ And the properties $\mathrm{S}_{1}(\Gamma, \Gamma)$ and $\mathrm{S}_{1}(\Omega, \Omega)$}

We already mentioned that the properties $S_{1}(\Gamma, \Gamma)$ and $\left(\begin{array}{l}\Omega \\ \Gamma\end{array}\right)$ were considered in the context of local properties of functions spaces. This is also the case for property $\mathrm{S}_{1}(\Omega, \Omega)$. A space $Y$ has countable strong fan tightness [19] if for each point $y \in Y$ and each sequence $A_{1}, A_{2}, \ldots$ of subsets of the space $Y$ with $y \in \bigcap_{n} \overline{A_{n}}$, there are points $a_{1} \in A_{1}, a_{2} \in A_{2}, \ldots$ such that $y \in \overline{\left\{a_{n}: n \in \mathbb{N}\right\}}$. For a set of reals $X$, the space $\mathrm{C}_{\mathrm{p}}(X)$ has countable strong fan tightness if and only if the set $X$ satisfies $\mathrm{S}_{1}(\Omega, \Omega)[19]$.

Let $\mathrm{O}$ be the class of all open covers of spaces. A space $X$ satisfies Menger's property $\mathrm{S}_{\text {fin }}(\mathrm{O}, \mathrm{O})$ if for each sequence $\mathcal{U}_{1}, \mathcal{U}_{2}, \ldots \in \mathrm{O}(X)$, there are finite sets $\mathcal{F}_{1} \subseteq \mathcal{U}_{1}, \mathcal{F}_{2} \subseteq \mathcal{U}_{2}, \ldots$ such that $\bigcup_{n} \mathcal{F}_{n} \in \mathrm{O}(X)$. In this section, we consider products of sets satisfying $\left(\begin{array}{l}\Omega \\ \Gamma\end{array}\right)$ and their relations to the properties $S_{1}(\Gamma, \Gamma), S_{1}(\Omega, \Omega)$ and $S_{\text {fin }}(O, O)$. We have the following implications between considered properties.

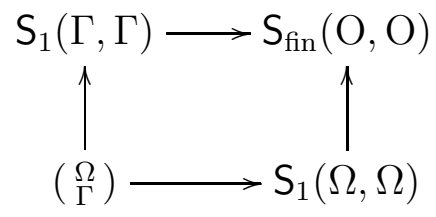

The properties $S_{1}(\Gamma, \Gamma)$ and $S_{1}(\Omega, \Omega)$ are much stronger than $S_{\text {fin }}(O, O)$. Indeed, all sets of reals satisfying $S_{1}(\Gamma, \Gamma)$ or $S_{1}(\Omega, \Omega)$ are totally imperfect [12, Theorem 2.3], i.e., they do not contain uncountable compact subsets; each compact set satisfies $\mathrm{S}_{\text {fin }}(\mathrm{O}, \mathrm{O})$. The existence of a nontrivial set of reals satisfying $\mathrm{S}_{1}(\Omega, \Omega)$ is independent of $Z F C$ : In the Laver model all sets of reals satisfying $S_{1}(\Omega, \Omega)$ are countable( [23, Theorem 17], [13]), and assuming that the Continuum Hypothesis holds, there is a nontrivial set satisfying $S_{1}(\Omega, \Omega)$ [12, Theorem 2.13]. In $\mathrm{ZFC}$, there is a nontrivial totally imperfect set satisfying $\mathrm{S}_{\mathrm{fin}}(\mathrm{O}, \mathrm{O})$ [4, Theorem 16].

Miller, Tsaban and Zdomskyy proved that there are two sets of reals satisfying $\left(\begin{array}{r}\Omega \\ \Gamma\end{array}\right)$ whose product space does not satisfy $\mathrm{S}_{\text {fin }}(\mathrm{O}, \mathrm{O})$ [16, Theorem 3.2.]. In Theorem 4.1, we gave a necessarily and sufficient condition when a $\mathfrak{p}$-generalized tower set is productively $\left(\begin{array}{r}\Omega \\ \Gamma\end{array}\right)$. Now, we show that the product space of a $\mathfrak{p}$-generalized tower set and a set satisfying $\left(\begin{array}{c}\Omega \\ \Gamma\end{array}\right)$, satisfies $\mathrm{S}_{1}(\Gamma, \Gamma)$ and $\mathrm{S}_{1}(\Omega, \Omega)$ in all finite powers (in fact, the property $\mathrm{S}_{1}(\Omega, \Omega$ ) is preserved by finite powers [19]).

Let $\mathbf{P}$ be a property of spaces. A space is productively $\mathbf{P}$, if its product space with any space satisfying $\mathbf{P}$, satisfies $\mathbf{P}$.

Theorem 5.1. Let $\kappa$ be an uncountable ordinal number such that each set of reals of cardinality less than $\kappa$ is productively $\mathrm{S}_{1}(\Gamma, \Gamma)$. The product space of finitely many $\kappa$-generalized tower sets and a set satisfying $\left(\begin{array}{l}\Omega \\ \Gamma\end{array}\right)$, satisfies $\mathrm{S}_{1}(\Gamma, \Gamma)$.

Let $X, Y$ be spaces and $m$ be a natural number. Identifying the space $X$ with $X \sqcup \emptyset$ and the space $Y$ with $\emptyset \sqcup Y$, the product space $X^{m} \times Y$ is a closed subset of $(X \sqcup Y)^{m+1}$.

Lemma 5.2. Let $X, Y \subseteq \mathrm{P}(\mathbb{N})$ and $m$ be a natural number. Let $\mathcal{U} \in \Omega\left(\operatorname{Fin}^{m} \times Y\right)$ be a family of open sets in $\mathrm{P}(\mathbb{N})^{m+1}$. There is a family $\mathcal{V} \in \Omega($ Fin $\sqcup Y)$ of open sets in $\mathrm{P}(\mathbb{N}) \sqcup \mathrm{P}(\mathbb{N})$ such that the family $\left\{V^{m+1} \cap\left(\mathrm{P}(\mathbb{N})^{m} \times Y\right): V \in \mathcal{V}\right\}$ refines the family $\mathcal{U}$. 
Proof. There are families $\mathcal{W}$ and $\mathcal{W}^{\prime}$ of open sets in $\mathrm{P}(\mathbb{N})$ such that the family

$$
\left\{W^{m} \times W^{\prime}: W \in \mathcal{W}, W^{\prime} \in \mathcal{W}^{\prime}\right\} \in \Omega\left(\operatorname{Fin}^{m} \times Y\right)
$$

refines the family $\mathcal{U}$. We have

$$
\left\{W \sqcup W^{\prime}: W \in \mathcal{W}, W^{\prime} \in \mathcal{W}^{\prime}\right\} \in \Omega(\text { Fin } \sqcup Y),
$$

and

$$
W^{m} \times W^{\prime}=\left(W \sqcup W^{\prime}\right)^{m+1} \cap\left(\mathrm{P}(\mathbb{N})^{m} \times Y\right)
$$

for all sets $W \in \mathcal{W}, W^{\prime} \in \mathcal{W}^{\prime}$.

Lemma 5.3. Let $\kappa$ be an uncountable ordinal number. Let $X \subseteq[\mathbb{N}]^{\infty}$ be a $\kappa$-generalized tower and $Y$ be a set satisfying $\left(\begin{array}{c}\Omega \\ \Gamma\end{array}\right)$. For each family $\mathcal{U} \in \Omega($ Fin $\sqcup Y)$ of open sets in $\mathrm{P}(\mathbb{N}) \sqcup \mathrm{P}(\mathbb{N})$, there are a set $S \subseteq X$ with $|S|<\kappa$ and a subfamily $\mathcal{V}$ of the family $\mathcal{U}$ with $\mathcal{V} \in \Gamma((X \backslash S) \sqcup Y)$.

Proof. Let $\mathcal{U} \in \Omega($ Fin $\sqcup Y)$ be a family of open sets in $\mathrm{P}(\mathbb{N}) \sqcup \mathrm{P}(\mathbb{N})$. The space Fin $\sqcup Y$ satisfies $\left(\begin{array}{l}\Omega \\ \Gamma\end{array}\right)$, and thus there is a subfamily $\mathcal{U}^{\prime}$ of the family $\mathcal{U}$ with $\mathcal{U}^{\prime} \in \Gamma($ Fin $\sqcup Y)$. Apply Lemma 4.2 to the family $\mathcal{U}^{\prime}$. Then there are a function $a \in[\mathbb{N}]^{\infty}$ and sets $U_{1}, U_{2}, \ldots \in \mathcal{U}^{\prime}$ such that for each set $x \in[\mathbb{N}]^{\infty}$ and all natural numbers $n$ :

$$
\text { If } x \cap[a(n), a(n+1))=\emptyset \text {, then } x \in U_{n} .
$$

Since $X$ is a $\kappa$-generalized tower, there are a set $b \in[\mathbb{N}]^{\infty}$ and a set $S \subseteq X$ with $|S|<\kappa$ such that

$$
x \cap \bigcup_{n \in b}[a(n), a(n+1)) \in \text { Fin }
$$

for all sets $x \in X \backslash S$. We have $\left\{U_{n}: n \in b\right\} \in \Gamma(X \backslash S)$. Thus,

$$
\left\{U_{n}: n \in b\right\} \in \Gamma((X \backslash S) \sqcup Y) .
$$

Lemma 5.4. Let $\kappa$ be an uncountable ordinal number. Let $X \subseteq[\mathbb{N}]^{\infty}$ be a $\kappa$-generalized tower, $Y$ be a set satisfying $\left(\begin{array}{l}\Omega \\ \Gamma\end{array}\right)$, and $m$ be a natural number. For each sequence $\mathcal{U}_{1}, \mathcal{U}_{2}$, $\ldots \in \Omega\left(\operatorname{Fin}^{m} \times Y\right)$ of families of open sets in $\mathrm{P}(\mathbb{N})^{m+1}$, there are a set $S \subseteq X$ with $|S|<\kappa$ and sets $U_{1} \in \mathcal{U}_{1}, U_{2} \in \mathcal{U}_{2}, \ldots$ such that

$$
\left\{U_{n}: n \in \mathbb{N}\right\} \in \Gamma\left(((X \backslash S) \cup \operatorname{Fin})^{m} \times Y\right) .
$$

Proof. Fix a natural number $m$. We may assume that the family $\mathcal{U}_{n+1}$ refines the family $\mathcal{U}_{n}$ for all natural numbers $n$. By Lemma [5.2, there is a sequence $\mathcal{V}_{1}, \mathcal{V}_{2}, \ldots \in \Omega($ Fin $\sqcup Y)$ such that the family

$$
\left\{V^{m+1} \cap\left(X^{m} \times Y\right): V \in \mathcal{V}_{n}\right\}
$$

refines the family $\mathcal{U}_{n}$ for all natural numbers $n$. Since the set Fin $\sqcup Y$ satisfies $\left(\begin{array}{l}\Omega \\ \Gamma\end{array}\right)$ and the properties $\left(\begin{array}{c}\Omega \\ \Gamma\end{array}\right)$ and $\mathrm{S}_{1}(\Omega, \Gamma)$ are equivalent [9, Theorem 2], there are sets $V_{1} \in \mathcal{V}_{1}, V_{2} \in \mathcal{V}_{2}$, ... such that $\left\{V_{n}: n \in \mathbb{N}\right\} \in \Gamma($ Fin $\sqcup Y)$. By Lemma 5.3, there are a set $a \in[\mathbb{N}]^{\infty}$ and a set $S \subseteq X$ with $|S|<\kappa$ such that $\left\{V_{n}: n \in a\right\} \in \Gamma(((X \backslash S) \cup$ Fin $) \sqcup Y)$. For each natural number $n \in a$, there is a set $U_{n} \in \mathcal{U}_{n}$ such that $V^{m+1} \cap\left(X^{m} \times Y\right) \subseteq U_{n}$. For each natural number $n \in a^{\mathrm{c}}$, there is a set $U_{n} \in \mathcal{U}_{n}$ such that $U_{n} \supseteq U_{k}$ for some natural number $k \in a$ with $n<k$. We have $\left\{U_{n}: n \in \mathbb{N}\right\} \in \Gamma\left(((X \backslash S) \cup \text { Fin })^{m} \times Y\right)$. 
Proof of Theorem 5.1. Let $Y$ be a set satisfying $\left(\begin{array}{c}\Omega \\ \Gamma\end{array}\right)$.

Let $X \subseteq[\mathbb{N}]^{\infty}$ be a $\kappa$-generalized and $\mathcal{U}_{1}, \mathcal{U}_{2}, \ldots \in \Gamma((X \cup$ Fin $) \times Y)$ be families of open sets in $\mathrm{P}(\mathbb{N})^{2}$. By Lemma [5.4, there are sets $U_{1} \in \mathcal{U}_{1}, U_{2} \in \mathcal{U}_{2}, \ldots$ and a set $S \subseteq X$ with $|S|<\kappa$ such that

$$
\left\{U_{n}: n \in \mathbb{N}\right\} \in \Gamma(((X \backslash S) \cup \text { Fin }) \times Y) .
$$

Since $|S|<\kappa$ and, by the assumption, each set of cardinality smaller than $\kappa$ is productively $\mathrm{S}_{1}(\Gamma, \Gamma)$, the product space $(S \cup$ Fin $) \times Y$ satisfies $\mathrm{S}_{1}(\Gamma, \Gamma)$. By Lemma 3.3, the product space $(X \cup$ Fin $) \times Y$ satisfies $\mathrm{S}_{1}(\Gamma, \Gamma)$.

Fix a natural number $m>1$ and assume that the statement is true for $m-1 \kappa$-generalized tower sets. Let $X_{1}, \ldots, X_{m} \subseteq[\mathbb{N}]^{\infty}$ be $\kappa$-generalized tower sets and

$$
\mathcal{U}_{1}, \mathcal{U}_{2}, \ldots \in \Gamma\left(\left(X_{1} \cup \text { Fin }\right) \times \cdots \times\left(X_{m} \cup \text { Fin }\right) \times Y\right)
$$

be families of open sets in $\mathrm{P}(\mathbb{N})^{m+1}$. By Lemma 2.3(1), the set $X:=\bigcup_{i \leq m} X_{i}$ is a $\kappa$ generalized tower. By Lemma 5.4 , there are sets $U_{1} \in \mathcal{U}_{1}, U_{2} \in \mathcal{U}_{2}, \ldots$ and a set $S \subseteq X$ with $|S|<\kappa$ such that

$$
\left\{U_{n}: n \in \mathbb{N}\right\} \in \Gamma\left(((X \backslash S) \cup \text { Fin })^{m} \times Y\right) .
$$

The set

$$
Z:=\left(\left(X_{1} \cup \text { Fin }\right) \times \cdots \times\left(X_{m} \cup \text { Fin }\right) \times Y\right) \backslash\left(\left((X \backslash S) \cup \text { Fin }^{m} \times Y\right)\right.
$$

satisfies $\mathrm{S}_{1}(\Gamma, \Gamma)$ : Fix a natural number $i \leq m$. By the inductive assumption, the product space

$$
\prod_{\substack{j \leq m \\ j \neq i}}\left(X_{j} \cup \text { Fin }\right) \times Y
$$

satisfies $S_{1}(\Gamma, \Gamma)$. By the assumption, each set of cardinality smaller than $\kappa$ is productively $\mathrm{S}_{1}(\Gamma, \Gamma)$. Since $\left|X_{i} \cap S\right|<\kappa$, the product space

$$
\prod_{\substack{j \leq m \\ j \neq i}}\left(X_{j} \cup \text { Fin }\right) \times\left(X_{i} \cap S\right) \times Y
$$

satisfies $S_{1}(\Gamma, \Gamma)$. A finite union of spaces satisfying $S_{1}(\Gamma, \Gamma)$, satisfies $S_{1}(\Gamma, \Gamma)$ [24, Theorem 5], and thus the set

$$
Z=\bigcup_{i \leq m} \prod_{\substack{j \leq m \\ j \neq i}}\left(X_{j} \cup \text { Fin }\right) \times\left(X_{i} \cap S\right) \times Y
$$

satisfies $\mathrm{S}_{1}(\Gamma, \Gamma)$, too.

By Lemma 3.3, the product space $\left(X_{1} \cup\right.$ Fin $) \times \cdots \times\left(X_{m} \cup\right.$ Fin $) \times Y$ satisfies $\mathrm{S}_{1}(\Gamma, \Gamma)$.

The property $\left(\begin{array}{l}\Omega \\ \Gamma\end{array}\right)$ is preserved by finite powers and each set of cardinality less than $\mathfrak{p}$ is productively $S_{1}(\Gamma, \Gamma)$ [5, Proposition 6.8], [24, Theorem 5]. Thus, we have the following result.

Corollary 5.5. The product space of a $\mathfrak{p}$-generalized tower set and a set satisfying $\left(\begin{array}{c}\Omega \\ \Gamma\end{array}\right)$, satisfies $\mathrm{S}_{1}(\Gamma, \Gamma)$ in all finite powers.

A set $X \subseteq \mathbb{N}^{\mathbb{N}}$ is guessable if there is a function $a \in \mathbb{N}^{\mathbb{N}}$ such that the sets $\{n: a(n)=x(n)\}$ are infinite, for all functions $x \in X$. Let $\operatorname{cov}(\mathcal{M})$ be the minimal cardinality of a family of meager subsets of the Baire space $\mathbb{N}^{\mathbb{N}}$, that covers $\mathbb{N}^{\mathbb{N}}$. The minimal cardinality of a subset of $\mathbb{N}^{\mathbb{N}}$ that is no guessable, is equal to $\operatorname{cov}(\mathcal{M})$ [5, Theorem 5.9]. 
Theorem 5.6. Let $\kappa$ be an uncountable ordinal number with $\kappa \leq \operatorname{cov}(\mathcal{M})$ such that $\kappa$ is regular or $\kappa<\operatorname{cov}(\mathcal{M})$. The product space of finitely many $\kappa$-generalized tower sets and a set satisfying $\left(\begin{array}{l}\Omega \\ \Gamma\end{array}\right)$, satisfies $\mathrm{S}_{1}(\Omega, \Omega)$.

We need the following Lemma.

Lemma 5.7. A union of less than $\operatorname{cov}(\mathcal{M})$ sets satisfying $\left(\begin{array}{r}\Omega \\ \Gamma\end{array}\right)$, satisfies $\mathrm{S}_{1}(\Omega, \mathrm{O})$.

Proof. Fix an ordinal number $\lambda<\operatorname{cov}(\mathcal{M})$. Let $X:=\bigcup_{\alpha<\lambda} X_{\alpha}$ be a union of sets satisfying $\left(\begin{array}{l}\Omega \\ \Gamma\end{array}\right)$ and $\mathcal{U}_{1}, \mathcal{U}_{2}, \ldots \in \Omega(X)$. Assume that $\mathcal{U}_{n}=\left\{U_{m}^{(n)}: m \in \mathbb{N}\right\}$ for all natural numbers $n$. For each ordinal number $\alpha<\lambda$, there is a function $f_{\alpha} \in \mathbb{N}^{\mathbb{N}}$ such that $\left\{U_{f_{\alpha}(n)}^{n}: n \in \mathbb{N}\right\} \in$ $\Gamma\left(X_{\alpha}\right)$. Since $\lambda<\operatorname{cov}(\mathcal{M})$, the set $\left\{f_{\alpha}: \alpha<\kappa\right\}$ is guessable. Then there is a function $g \in \mathbb{N}^{\mathbb{N}}$ such that the sets $\left\{n: f_{\alpha}(n)=g(n)\right\}$ are infinite, for all ordinal numbers $\alpha<\kappa$. Thus, $\left\{U_{g(n)}^{(n)}: n \in \mathbb{N}\right\} \in \mathrm{O}(X)$.

Proof of Theorem 5.6. A set of reals satisfies $\mathrm{S}_{1}(\Omega, \Omega)$ if and only if it satisfies $\mathrm{S}_{1}(\mathrm{O}, \mathrm{O})$ in all finite powers [19]. The properties $\mathrm{S}_{1}(\mathrm{O}, \mathrm{O})$ and $\mathrm{S}_{1}(\Omega, \mathrm{O})$ are equivalent [23, Theorem 17] and property $\left(\begin{array}{l}\Omega \\ \Gamma\end{array}\right)$ is preserved by finite powers. Thus, it is enough to show that the statement is true, when consider the property $\mathrm{S}_{1}(\Omega, \mathrm{O})$ instead of $\mathrm{S}_{1}(\Omega, \Omega)$. We prove a formally stronger assertion that the product space of finitely many $\kappa$-generalized tower sets, a set satisfying $\left(\begin{array}{c}\Omega \\ \Gamma\end{array}\right)$ and a set of cardinality less than $\operatorname{cov}(\mathcal{M})$, satisfies $S_{1}(\Omega, O)$.

Let $Y$ be a set satisfying $\left(\begin{array}{l}\Omega \\ \Gamma\end{array}\right)$ and $Z$ be a set with $|Z|<\operatorname{cov}(\mathcal{M})$.

Let $X \subseteq[\mathbb{N}]^{\infty}$ be a $\kappa$-generalized tower and $\mathcal{U}_{1}, \mathcal{U}_{2}, \ldots \in \Omega((X \cup$ Fin $) \times Y \times Z)$ be families of open sets in $\mathrm{P}(\mathbb{N})^{3}$, where $\mathcal{U}_{n}=\left\{U_{m}^{(n)}: m \in \mathbb{N}\right\}$ for all natural numbers $n$. Fix a point $z \in Z$. By Lemma 5.4, there are a function $g_{z} \in \mathbb{N}^{\mathbb{N}}$ and a set $S_{z} \subseteq X$ with $\left|S_{z}\right|<\kappa$ such that

$$
\left\{U_{g_{z}(n)}^{(n)}: n \in \mathbb{N}\right\} \in \Gamma\left(\left(\left(X \backslash S_{z}\right) \cup \operatorname{Fin}\right) \times Y \times\{z\}\right)
$$

Since $|Z|<\operatorname{cov}(\mathcal{M})$, there is a function $g \in \mathbb{N}^{\mathbb{N}}$ such that the sets $\left\{n: g(n)=g_{z}(n)\right\}$ are infinite for all points $z \in Z$. Let $S:=\bigcup\left\{S_{z}: z \in Z\right\}$. We have

$$
\left\{U_{g(n)}^{(n)}: n \in \mathbb{N}\right\} \in \Gamma(((X \backslash S) \cup \text { Fin }) \times Y \times Z)
$$

Then there are sets $U_{1} \in \mathcal{U}_{1}, U_{3} \in \mathcal{U}_{3}, \ldots$ such that

$$
\left\{U_{2 n-1}: n \in \mathbb{N}\right\} \in \Gamma(((X \backslash S) \cup \text { Fin }) \times Y \times Z) .
$$

By the assumption about the ordinal number $\kappa$, we have $|S|<\operatorname{cov}(\mathcal{M})$. Thus, $|S \times Z|<$ $\operatorname{cov}(\mathcal{M})$. By Lemma 5.7, the product space $S \times Y \times Z$ satisfies $\mathrm{S}_{1}(\Omega, \mathrm{O})$. There are sets $U_{2} \in \mathcal{U}_{2}, U_{4} \in \mathcal{U}_{4}, \ldots$ such that

$$
\left\{U_{2 n}: n \in \mathbb{N}\right\} \in \mathrm{O}(S \times Y \times Z) .
$$

Finally, we have

$$
\left\{U_{n}: n \in \mathbb{N}\right\} \in \mathrm{O}((X \cup \text { Fin }) \times Y \times Z) .
$$

Fix a natural number $m>1$ and assume that the statement is true for $m-1 \kappa$-generalized tower sets. Let $X_{1}, \ldots, X_{m} \subseteq[\mathbb{N}]^{\infty}$ be $\kappa$-generalized towers. Let

$$
\mathcal{U}_{1}, \mathcal{U}_{2}, \ldots \in \Omega\left(\left(X_{1} \cup \text { Fin }\right) \times\left(X_{m} \cup \text { Fin }\right) \times Y \times Z\right)
$$

be a sequence of families of open sets in $\mathrm{P}(\mathbb{N})^{m+2}$, where $\mathcal{U}_{n}=\left\{U_{m}^{(n)}: m \in \mathbb{N}\right\}$ for all natural numbers $n$. By Lemma 2.3(1), the set $X:=\bigcup_{i \leq m} X_{i}$ is a $\kappa$-generalized tower. Fix a point 
$z \in Z$. By Lemma 5.4, there are a function $g_{z} \in \mathbb{N}^{\mathbb{N}}$ and a set $S_{z} \subseteq X$ with $\left|S_{z}\right|<\kappa$ such that

$$
\left\{U_{g_{z}(n)}^{(n)}: n \in \mathbb{N}\right\} \in \Gamma\left(\left(\left(X \backslash S_{z}\right) \cup \text { Fin }\right)^{m} \times Y \times\{z\}\right) .
$$

Since $|Z|<\operatorname{cov}(\mathcal{M})$, there is a function $g \in \mathbb{N}^{\mathbb{N}}$ such that the sets $\left\{n: g(n)=g_{z}(n)\right\}$ are infinite for all points $z \in Z$. Let $S:=\bigcup\left\{S_{z}: z \in Z\right\}$. We have

$$
\left\{U_{g(n)}^{(n)}: n \in \mathbb{N}\right\} \in \Gamma\left(((X \backslash S) \cup \text { Fin })^{m} \times Y \times Z\right) .
$$

Then there are sets $U_{1} \in \mathcal{U}_{1}, U_{3} \in \mathcal{U}_{3}, \ldots$ such that

$$
\left\{U_{2 n-1}: n \in \mathbb{N}\right\} \in \Gamma\left(((X \backslash S) \cup \text { Fin })^{m} \times Y \times Z\right) .
$$

The set

$$
T:=\left(\left(X_{1} \cup \text { Fin }\right) \times \cdots \times\left(X_{m} \cup \text { Fin }\right) \times Y \times Z\right) \backslash\left(((X \backslash S) \cup \text { Fin })^{m} \times Y \times Z\right)
$$

satisfies $\mathrm{S}_{1}(\Omega, \mathrm{O})$ : Fix a natural number $i \leq m$. By the inductive assumption, the product space

$$
\prod_{\substack{j \leq m \\ j \neq i}}\left(X_{j} \cup \text { Fin }\right) \times Y \times Z
$$

satisfies $\mathrm{S}_{1}(\Omega, \mathrm{O})$. By the assumption about the ordinal number $\kappa$, we have $|S|<\operatorname{cov}(\mathcal{M})$. Since $\left|X_{i} \cap S\right|<\operatorname{cov}(\mathcal{M})$, we have $\left|\left(X_{i} \cap S\right) \times Z\right|<\operatorname{cov}(\mathcal{M})$. By the inductive assumption, the product space

$$
\prod_{\substack{j \leq m \\ j \neq i}}\left(X_{j} \cup \text { Fin }\right) \times Y \times\left(X_{i} \cap S\right) \times Z
$$

satisfies $\mathrm{S}_{1}(\Omega, \mathrm{O})$. A finite union of sets satisfying $\mathrm{S}_{1}(\Omega, \mathrm{O})$, satisfies $\mathrm{S}_{1}(\Omega, \mathrm{O})$ [3, Theorem 2.3.9], and thus the set

$$
T=\bigcup_{i \leq m} \prod_{\substack{j \leq m \\ j \neq i}}\left(X_{j} \cup \text { Fin }\right) \times Y \times\left(X_{i} \cap S\right) \times Z,
$$

satisfies $\mathrm{S}_{1}(\Omega, \mathrm{O})$, too. There are sets $U_{2} \in \mathcal{U}_{2}, U_{4} \in \mathcal{U}_{4}, \ldots$ such that

$$
\left\{U_{2 n}: n \in \mathbb{N}\right\} \in \mathrm{O}(T)
$$

Finally, we have

$$
\left\{U_{n}: n \in \mathbb{N}\right\} \in \mathrm{O}\left(\left(X_{1} \cup \text { Fin }\right) \times \cdots \times\left(X_{m} \cup \text { Fin }\right) \times Y \times Z\right) .
$$

Corollary 5.8. Let $\kappa$ be an uncountable ordinal number with $\kappa \leq \operatorname{cov}(\mathcal{M})$ such that $\kappa$ is regular or $\kappa<\operatorname{cov}(\mathcal{M})$. Each $\kappa$-generalized tower set satisfies $\mathrm{S}_{1}(\Omega, \Omega)$.

Since the ordinal number $\mathfrak{p}$ is regular and $\mathfrak{p} \leq \operatorname{cov}(\mathcal{M})$, we have the following result.

Corollary 5.9. The product space of a $\mathfrak{p}$-generalized tower set and a set satisfying $\left(\begin{array}{l}\Omega \\ \Gamma\end{array}\right)$, satisfies $\mathrm{S}_{1}(\Omega, \Omega)$. 


\section{REMARKS AND OPEN PROBLEMS}

6.1. Around Scheepers's Conjecture. A clopen cover of a space is a cover whose members are clopen subsets of the space. Let $\Gamma_{\text {clp }}$ be the class of all clopen $\gamma$-covers of spaces. A set of reals is a wQN-space if and only if it satisfies $S_{1}\left(\Gamma_{\text {clp }}, \Gamma_{\text {clp }}\right)$ [6, Theorem 9]. The following conjecture was formulated by Scheepers.

Conjecture 6.1 ([22]). The properties $\mathrm{S}_{1}(\Gamma, \Gamma)$ and $\mathrm{S}_{1}\left(\Gamma_{\mathrm{clp}}, \Gamma_{\mathrm{clp}}\right)$ are equivalent.

The property $S_{1}(\Gamma, \Gamma)$ describes a local property of functions spaces: Let $\mathbb{R}$ be the real line with the usual topology. Let $X$ be a set of reals. A function $f: X \rightarrow \mathbb{R}$ is upper semicontinuous if the sets $\{x \in X: f(x)<a\}$ are open for all real numbers $a$. By the result of Bukovský [7, Theorem 13], the set $X$ satisfies $\mathrm{S}_{1}(\Gamma, \Gamma)$ if and only if it is an $S P P^{*}$ space, that is, for each sequence $\left\langle f_{1, m}\right\rangle_{m \in \mathbb{N}},\left\langle f_{2, m}\right\rangle_{m \in \mathbb{N}}, \ldots$ of sequences of upper continuous functions on $X$, each of them converging pointwise to the constant zero function $\mathbf{0}$, there is a sequence $\left\langle m_{n}\right\rangle_{n \in \mathbb{N}}$ of natural numbers such that the sequence $\left\langle f_{n, m_{n}}\right\rangle_{n \in \mathbb{N}}$ converges to $\mathbf{0}$. Thus, in the language of functions spaces, the above Scheepers conjecture asks whether the properties wQN and $\mathrm{SSP}^{*}$ are equivalent.

6.2. Generalized towers and productivity of $\bigcup_{\text {fin }}(\mathcal{O}, \Gamma)$. A space $X$ satisfies Hurewicz's property $\mathrm{U}_{\text {fin }}(\mathcal{O}, \Gamma)$ if for each sequence $\mathcal{U}_{1}, \mathcal{U}_{2}, \ldots \in \mathrm{O}(X)$ there are finite sets $\mathcal{F}_{1} \subseteq \mathcal{U}_{1}, \mathcal{F}_{2} \subseteq$ $\mathcal{U}_{2}, \ldots$ such that $\left\{\bigcup \mathcal{F}_{n}: n \in \mathbb{N}\right\} \in \Gamma(X)$. We have the following implications between considered properties

$$
\mathrm{S}_{1}(\Gamma, \Gamma) \longrightarrow \mathrm{U}_{\mathrm{fin}}(\mathcal{O}, \Gamma) \longrightarrow \mathrm{S}_{\text {fin }}(\mathrm{O}, \mathrm{O})
$$

and the property $\mathrm{U}_{\text {fin }}(\mathcal{O}, \Gamma)$ is strictly in between properties $\mathrm{S}_{1}(\Gamma, \Gamma)$ and $\mathrm{S}_{\text {fin }}(\mathrm{O}, \mathrm{O})([12$, Theorems 2.2, 2.4], [30, Theorem 3.9]). Let $\mathrm{cF}$ be the set of all cofinite subsets of $\mathbb{N}$. For functions $z, t \in[\mathbb{N}]^{\infty}$, we write $z \leq^{\infty} t$ if $t \mathbb{Z}^{*} z$.

Definition 6.2 ([27, Definition 4.1]). A set $X \subseteq[\mathbb{N}]^{\infty}$ is a cF-scale if for each element $z \in[\mathbb{N}]^{\infty}$, there is an element $t \in[\mathbb{N}]^{\infty}$ such that

$$
z \leq^{\infty} t \leqslant{ }^{*} x
$$

for all but less than $\mathfrak{b}$ functions $x \in X$.

Lemma 6.3. Each $\mathfrak{b}$-generalized tower is a $\mathrm{cF}$-scale.

Proof. Let $X \subseteq[\mathbb{N}]^{\infty}$ be a $\mathfrak{b}$-generalized tower and $z \in[\mathbb{N}]^{\infty}$ be an element such that $z(1) \neq 1$. Define an element $\tilde{z}$ such that $\tilde{z}(1):=z(1)$, and $\tilde{z}(n+1):=z(\tilde{z}(n))$ for all natural numbers $n$. There is a set $b \in[\mathbb{N}]^{\infty}$ such that

$$
x \cap \bigcup_{n \in b}[\tilde{z}(n), \tilde{z}(n+1)) \in \text { Fin }
$$

for all but less than $\mathfrak{b}$ many elements $x \in X$. We have $b^{\mathrm{c}} \in[\mathbb{N}]^{\infty}$. Then the set $t:=$ $\bigcup_{n \in b^{c}}[\tilde{z}(n), \tilde{z}(n+1))$ omits infinitely many intervals $[\tilde{z}(n), \tilde{z}(n+1))$ and $x \subseteq^{*} t$ for all but less than $\mathfrak{b}$ elements $x \in X$. We have

$$
z(\tilde{z}(n)) \leq \tilde{z}(n+1) \leq t(\tilde{z}(n))
$$

for all natural numbers $n \in b^{c}$. Thus, $z \leqslant^{\infty} t$. For an element $x \in[\mathbb{N}]^{\infty}$ such $x \subseteq^{*} t$ and $t \backslash x \in[\mathbb{N}]^{\infty}$, we have $t \leq^{*} x$. There are only countably many elements $x \in[\mathbb{N}]^{\infty}$ with $t \backslash x \in$ Fin. Thus,

$$
z \leq^{\infty} t \leq^{*} x
$$


for all but less than $\mathfrak{b}$ elements $x \in X$.

By the result of Tsaban and the first named author [27, Theorem 5.4], we have the following corollary.

Corollary 6.4. Let $\kappa$ be an uncountable ordinal number with $\kappa \leq \mathfrak{b}$. Each $\kappa$-generalized tower set is productively $\mathrm{U}_{\text {fin }}(\mathcal{O}, \Gamma)$.

\subsection{Questions.}

Problem 6.5. Is a $\mathfrak{b}$-unbounded tower, provably, productively $\mathrm{S}_{1}(\Gamma, \Gamma)$ ? Is this the case assuming the Continuum Hypothesis?

Problem 6.6. Assume Martin Axiom and the negation of the Continuum Hypothesis. Is each set of cardinality less than $\mathfrak{c}$ productively $\left(\begin{array}{l}\Omega \\ \Gamma\end{array}\right)$ ?

Problem 6.7. Is it consistent that $\mathfrak{p}>\omega_{1}$, each set of cardinality less than $\mathfrak{p}$ is productively $\left(\begin{array}{c}\Omega \\ \Gamma\end{array}\right)$ and there is a $\mathfrak{p}$-generalized tower?

Problem 6.8. Let $\kappa$ be an uncountable ordinal number. Does the existence of a $\kappa$-generalized tower imply the existence of a $\kappa$-unbounded tower?

Problem 6.9. Let $\kappa$ be an uncountable ordinal number. Is a union of less than $\mathfrak{b}$ many $\kappa$-generalized towers, a $\kappa$-generalized tower?

\section{REFERENCES}

[1] A. Arhangel'skiŭ, The frequency spectrum of a topological space and the classification of spaces, Soviet Math. Dokl. 13 (1972), 1185-1189.

[2] A. Arhangel'skiı̌, Hurewicz spaces, analytic sets and fan tightness of function spaces, Soviet Mathematics Doklady 33 (1986), 396-399.

[3] T. Bartoszyński, H. Judah, Set Theory: On the structure of the real line, A. K. Peters, Massachusetts: 1995.

[4] T. Bartoszyński, B. Tsaban, Hereditary topological diagonalizations and the Menger-Hurewicz Conjectures, Proceedings of the American Mathematical Society 134 (2006), 605-615.

[5] A. Blass, Combinatorial cardinal characteristics of the continuum, in: Handbook of Set Theory (M. Foreman, A. Kanamori, eds.), Springer, 2010, 395-489.

[6] L. Bukovský, J. Haleš, QN-spaces, wQN-spaces and covering properties, Topology and its Applications 154 (2007), 848-858.

[7] L. Bukovský, On $\mathrm{wQN}_{*}$ and wQN* spaces, Topology and its Applications 156 (2008), 24-27.

[8] L. Bukovský, I. Recław, M. Repický, Spaces not distinguishing convergences of real-valued functions, Topology and its Applications 112 (2001), 13-40.

[9] J. Gerlits, Zs. Nagy, Some properties of $\mathrm{C}_{\mathrm{p}}(X)$, I, Topology and its Applications 14 (1982), 151-161.

[10] F. Galvin, A. Miller, $\gamma$-sets and other singular sets of real numbers, Topology and its Applications 17 (1984), 145-155.

[11] J. Haleš, On Scheepers' conjecture, Acta Universitatis Carolinae. Mathematica et Physica 46 (2005), $27-31$.

[12] W. Just, A. Miller, M. Scheepers, P. Szeptycki, The combinatorics of open covers II, Topology and its Applications 73 (1996), 241-266.

[13] R. Laver, On the consistency of Borel's conjecture, Acta Mathematicae 137 (1976), 151-169.

[14] A. Miller, A hodgepodge of sets of reals, Note di Matematica 27 (2007), suppl. 1, 25-39.

[15] A. Miller, B. Tsaban, Point-cofinite covers in Laver's model, Proceedings of the American Mathematical Society 138 (2010), 3313-3321.

[16] A. Miller, B. Tsaban, L. Zdomskyy, Selective covering properties of product spaces, II: $\gamma$ spaces, Transactions of the American Mathematical Society 368 (2016), 2865-2889. 
[17] T. Orenshtein, B. Tsaban, Linear $\sigma$-additivity and some applications, Transactions of the American Mathematical Society 363 (2011), 3621-3637.

[18] A. Osipov, P. Szewczak, B. Tsaban, Strongly sequentially separable function spaces, via selection principles, Topology and its Applications, 270 (2020), 106942.

[19] M. Sakai, Property C" and function spaces, Proceedings of the American Mathematical Society 104 (1988), 917-919.

[20] M. Sakai, The sequence selection properties of $\mathrm{C}_{\mathrm{p}}(X)$, Topology and its Applications 154 (2007), 552560.

[21] M. Sakai, Selection principles and upper semicontinuous functions, Colloquium Mathematicum 117 (2009), 251-256..

[22] M. Sakai, M. Scheepers, The combinatorics of open covers, in: Recent Progress in General Topology III (K. Hart, J. van Mill, P. Simon, eds.), Atlantis Press, 2014, 751-800.

[23] M. Scheepers, Combinatorics of open covers. I: Ramsey theory, Topology and its Applications 69 (1996), $31-62$.

[24] M. Scheepers, Sequential convergence in $\mathrm{C}_{\mathrm{p}}(X)$ and a covering property, East-West Journal of Mathematics 1 (1999), 207-214.

[25] M. Scheepers, $\mathrm{C}_{\mathrm{p}}(X)$ and Arhangel'skiu's $\alpha_{i}$ spaces, Topology and its Applications 89 (1998), 265-275.

[26] M. Scheepers, B. Tsaban, The combinatorics of Borel covers, Topology and its Applications 121 (2002), $357-382$.

[27] P. Szewczak, B. Tsaban, Products of Menger spaces: A combinatorial approach, Annals of Pure and Applied Logic 168 (2017), 1-18.

[28] B. Tsaban, Additivity numbers of covering properties, in: Selection Principles and Covering Properties in Topology (L. Kocinac, editor), Quaderni di Matematica 18, Seconda Universita di Napoli, Caserta 2006, 245-282.

[29] B. Tsaban, Menger's and Hurewicz's Problems: Solutions from "The Book" and refinements, Contemporary Mathematics 533 (2011), 211-226.

[30] B. Tsaban, L. Zdomskyy, Scales, fields, and a problem of Hurewicz, Journal of the European Mathematical Society 10 (2008), 837-866.

[31] B. Tsaban, L. Zdomskyy, Hereditarily Hurewicz spaces and Arhangel'skiu sheaf amalgamations, Journal of the European Mathematical Society 12 (2012), 353-372.

Piotr Szewczak, Institute of Mathematics, Faculty of Mathematics and Natural Science College of Sciences, Cardinal Stefan Wyszyński University in WarsaW, Wóycickiego 1/3, 01-938 WarsaW, Poland

E-mail address: p.szewczak@wp.pl

$U R L:$ http://piotrszewczak.pl

Magdalena Weudecka, Institute of Mathematics, Faculty of Mathematics and Natural Science College of Sciences, Cardinal Stefan Wyszyński University in WarsaW, Wóycickiego 1/3, 01-938 Warsaw, Poland

E-mail address: m.wludecka@gmail.com 